rials and molecular responses, through engineering metamaterials to a bio or chemical hazard of interest, will provide an interesting approach beyond simple dielectric induced resonance shifts."

STEVEN TROHALAKI

\section{Nonlinear Optical Mixing Enables Silicon-Chip-Based Ultrafast Oscilloscope with Sub-Picosecond Resolution}

As high-speed optical communications and ultrafast science have pushed the envelope on the meaning of "fast," they have created a corresponding need for ultrafast measurement technologies. Techniques based on nonlinear optical mixing and repeated averaging can achieve very high time resolutions, but are not useful for measuring single, nonperiodic, or asynchronous optical events. Now A.L. Gaeta, M. Lipson, and colleagues at Cornell University have developed a device that may lead to a new class of ultrafast oscilloscopes based on nonlinear optical mixing in silicon. Their device has a resolution of $220 \mathrm{fs}$ and a record length of 100 ps, and is fully compatible with complementary metal oxide semiconductor (CMOS) technology. They reported their results in the November 6, 2008 issue of Nature (DOI: 10.1038/nature07430; p. 81).

The research team's device uses the technique of time-to-frequency conversion, in which a quadratically varying phase shift is added to the optical signal to be measured. This phase shift causes the signal to evolve so that at a later period its amplitude in time is a scaled replica of its original frequency spectrum, and its frequency spectrum is a scaled replica of its original amplitude in time. The group accomplishes this phase shift addition by injecting the optical signal (centered at $1580 \mathrm{~nm}$ wavelength) into a $1.5-\mathrm{cm}$ length nanoscale silicon-on-insulator waveguide (with a cross-sectional area of $300 \mathrm{~nm}$ by $750 \mathrm{~nm}$ ) along with a suitably prepared pump signal wave. Four-wave mixing in the waveguide leads to a quadratic phase shift (or linear frequency shift) that is equivalent to $1 \mathrm{~nm}$ of wavelength shift for every 5.2 ps shift in time. After the waveguide and an appropriate signal propagation time, the signal spectrum is measured by an optical spectrometer, and the spectrum is scaled to obtain the original signal amplitude in time.

To characterize the device, the researchers first measured several 342-fs optical pulses with varying delays, determining that the device's record length is $100 \mathrm{ps}$ and its inherent resolution is $220 \mathrm{fs}$. These limits are likely caused by highorder dispersion in the optical fibers carry- ing the signal and the performance of the spectrometer, and not by the four-wave mixing in the silicon waveguide. The researchers next measured several more complicated signals, and compared the results with measurements of the same signals using an average of many conventional cross-correlation measurements. The results clearly demonstrate the accuracy of the device and its ability to maintain a long (100 ps) record with high time resolution in a single shot.

According to the researchers, the use of dispersion-flattened fiber or dispersionengineered waveguides may enable sub100-fs resolution, and the technique can be used with other CMOS-compatible waveguiding materials such as $\mathrm{SiN}$ or $\mathrm{SiON}$. Additionally, the individual components of the device are all the subject of extensive current research in photonics, suggesting that it may soon be possible to integrate the entire device on a chip. If that is correct, both telecommunications engineers and ultrafast scientists may one day think of bulky, super-picosecond oscilloscopes as a thing of the past.

\section{COLIN McCORMICK}

\section{Spin-Echo Technique in Nitrogen-Vacancy Diamond Impurities Enables Nanoscale Magnetic Sensing}

The ability to detect extremely weak magnetic fields at short distances would enable important applications in a wide range of fields, from probing individual nuclear spins in complex biological molecules to storing and controlling quantum information encoded in electronic or nuclear spins. In pursuit of this ability, researchers M.D. Lukin and A. Yacoby of Harvard University, R.L. Walsworth of Harvard and the Harvard-Smithsonian Center for Astrophysics, J.S. Hodges of Harvard and the Massachusetts Institute of Technology, J.M. Taylor of the Massachusetts Institute of Technology, M.V.G. Dutt of the University of Pittsburgh, and their colleagues have demonstrated the use of coherent control of an individual electronic spin in nitrogen-vacancy diamond impurities to detect magnetic fields at the nano-Tesla level. In combination with diamond nanocrystals, this technique may lead to a new class of sensitive, extremely short-range magnetic sensors.

Electronic spin in nitrogen-vacancy (NV) impurities in diamond has been extensively studied as a candidate quantum bit because of its relative isolation from environmental effects that would cause quantum decoherence. Since ${ }^{12} \mathrm{C}$ has no nuclear magnetic moment, the primary source of local magnetic field for NV impurities comes from the nuclear spin of the small number (roughly 1\% isotopic abundance) of ${ }^{13} \mathrm{C}$ atoms in the diamond lattice. As reported in October 2, 2008 issue of Nature (DOI: $10.1038 /$ nature07279; p. 644), the researchers used a standard spin-echo technique to manipulate the electronic spin of a single NV impurity in a bulk, ultrapure diamond sample. By matching the length of the spin-echo sequence to the period of the Larmor precession of the ${ }^{13} \mathrm{C}$ nuclei caused by an external dc magnetic field, the researchers were able to decouple the NV electronic spin from the nuclear magnetic field, and obtain strong spin-echo signals at times exceeding $0.5 \mathrm{~ms}$. They then imposed a weak ( 100 nT) ac magnetic field on the sample, and observed a sinusoidal variation of the decoupled spinecho signal as a function of the ac field strength, caused by the accumulation of additional magnetic-precession phase from the time-varying Zeeman shift of the NV electronic spin during the spin-echo sequence. The system achieved a resolution of a few nano-Teslas for a $3.2-\mathrm{kHz}$ ac magnetic field after $100 \mathrm{~s}$ of averaging, limited by the photon shot noise in the optical readout of the spin-echo signal.

The researchers also conducted a similar experiment using NV impurities in 30-nmdiameter diamond nanocrystals. These samples contained more spin impurities, leading to a shorter spin-coherence time (4-10 $\mu \mathrm{s})$, and the technique displayed a sensitivity of $0.5 \mu \mathrm{T} \mathrm{Hz}-1 / 2$ for a field at $380 \mathrm{kHz}$. A higher sample purity and higher efficiency optical detection would likely improve this sensitivity significantly. In related work, a group at Stuttgart and Texas A\&M Universities led by F. Jelezko, P. Hemmer, and J. Wrachtrup has used diamond nanocrystals to create a scanning magnetic-field sensor. In combination, these results may soon enable an extremely short-range ( 10 nm), highsensitivity $(\sim 1 \mathrm{nT})$ magnetic field sensor, with important applications in fields ranging from molecular biology to quantum information.

COLIN MCCORMICK

\section{Silicon Nitride Membrane Dynamic Masking Allows Improved Shapes of Near-Field Optical Apertures Fabricated by FIB}

Plasmonic devices structured on the scale of tens of nanometers for applications in optical interconnects, data storage, nearfield lithography, and bio-sensors are often produced by directly milling the metal surface with a focused ion beam (FIB) (direct metal milling, DMM). However, obtaining high-quality structures by using this technique is difficult, as the ion beam's 